\title{
UHPLC/MS-MS Analysis of Six Neonicotinoids in Honey by Modified QuEChERS: Method Development, Validation, and Uncertainty Measurement
}

\author{
Michele Proietto Galeano, Monica Scordino, Leonardo Sabatino, Valentina Pantò, \\ Giovanni Morabito, Elena Chiappara, Pasqualino Traulo, and Giacomo Gagliano
}

Dipartimento dell'Ispettorato Centrale della Tutela della Qualità e della Repressione Frodi dei Prodotti Agroalimentari (ICQRF), Laboratory of Catania, Ministero delle Politiche Agricole Alimentari e Forestali (MIPAAF), Via A. Volta 19, 95122 Catania, Italy

Correspondence should be addressed to Monica Scordino; m.scordino@mpaaf.gov.it

Received 9 January 2013; Accepted 8 March 2013

Academic Editor: Yao-wen Huang

Copyright (C) 2013 Michele Proietto Galeano et al. This is an open access article distributed under the Creative Commons Attribution License, which permits unrestricted use, distribution, and reproduction in any medium, provided the original work is properly cited.

Rapid and reliable multiresidue analytical methods were developed and validated for the determination of 6 neonicotinoids pesticides (acetamiprid, clothianidin, imidacloprid, nitenpyram, thiacloprid, and thiamethoxam) in honey. A modified QuEChERS method has allowed a very rapid and efficient single-step extraction, while the detection was performed by UHPLC/MS-MS. The recovery studies were carried out by spiking the samples at two concentration levels $(10$ and $40 \mu \mathrm{g} / \mathrm{kg})$. The methods were subjected to a thorough validation procedure. The mean recovery was in the range of 75 to $114 \%$ with repeatability below $20 \%$. The limits of detection were below $2.5 \mu \mathrm{g} / \mathrm{kg}$, while the limits of quantification did not exceed $4.0 \mu \mathrm{g} / \mathrm{kg}$. The total uncertainty was evaluated taking the main independent uncertainty sources under consideration. The expanded uncertainty did not exceed $49 \%$ for the $10 \mu \mathrm{g} / \mathrm{kg}$ concentration level and was in the range of $16-19 \%$ for the $40 \mu \mathrm{g} / \mathrm{kg}$ fortification level.

\section{Introduction}

Neonicotinoids are a relatively new class of insecticides that share a common mode of action that affect the central nervous system of insects, resulting in paralysis and death [1]. They possess either a nitromethylene, nitroimine, or cyanoimine group [2]. They include acetamiprid, clothianidin, imidacloprid, nitenpyram, thiacloprid, and thiamethoxam. Studies suggested that neonicotinoids residues can accumulate in pollen and nectar of treated plants and represent a potential risk to pollinators [3]. Therefore, neonicotinic pesticides may play a role in recent pollinator declines. The Honey Italian Observatory stated that in 2008 more than half of Italian hives, and that 600,000 of a total of 1,100,000 have been put out of production for the depopulation of entire apiaries. The honey production in 2008 fell by $50 \%$ reduced to 7,000 tons. One result might be expected given that the previous year, the European Food Safety Authority (EFSA) stated that the bee die-off had hit the $50 \%$ bee population, compared to the annual average of $15 \%$.

Neonicotinoids can also be persistent in the environment and, when used as seed treatments, translocate to residues in pollen and nectar of treated plants. The potential for these residues to affect bees and other pollinators remains uncertain. Despite these uncertainties, neonicotinoids are beginning to dominate the market place because of their high systemicity, the broad spectrum of action, and the reduced dose. In light of these findings, the Italian Ministry of Agriculture has asked the Ministry of Health to suspend action. The Ministry of Health, after consultation with the Pesticides Committee, issued the ministerial decree of September 17, 2008 that stated the precautionary suspension of the authorized use for the seeds tanning of plant protection products containing the active substances clothianidin, thiamethoxam, imidacloprid, and fipronil [4]. On June 25, 2012, a decree of the Ministry of Health extended to January 31, 2013 stating 
the neonicotinoids suspension for seeds treatment [5]. Similar measures have already been taken by other European states.

Recently, many researchers detected these insecticides in honey bees, honey, soil, pollen, and treated seeds for agriculture [6-12]. Measurement of pesticide residues in different matrices involves two basic steps, namely, sample preparation (extraction and clean up) and instrumental analysis. Ideally, a sample preparation should be rapid, simple, cheap, and environment friendly and provide clean extracts. After extraction, clean up is the most important process for multiresidue analysis. QuEChERS (Quick Easy Cheap Effective Rugged Safe) technique, which was developed between 2000 and 2002 and first reported in 2003 [13], is a fast and complete extraction and clean up procedure and also employs the use of dispersive-solid phase extraction (d-SPE) for sample clean up.

In this paper, we report a rapid modified QuEChERS method for multiresidue analysis for 6 neonicotinoids (acetamiprid, clothianidin, imidacloprid, nitenpyram, thiacloprid, and thiamethoxam) in honey with good selectivity, sensitivity, and cost effectiveness. In order to demonstrate the suitability of the method for routine regulatory purposes, the method was validated and the statistical parameters are discussed.

\section{Materials and Methods}

2.1. Reagents and Standards. The certified analytical standards of all the 6 pesticides (acetamiprid, clothianidin, imidacloprid, nitenpyram, thiacloprid, and thiamethoxam) and internal standard Tris(1-chloro-2-propyl)phosphate (TCPP) were purchased from Ultra Scientific (Bologna, Italy) (100.0 \pm $0.5 \mu \mathrm{g} / \mathrm{mL}$ each) in acetonitrile. All the solvents and chemicals used in the study were of analytical reagent (AR) grade, ethanol was supplied by Romil (Milan, Italy), and formic acid, ammonium formiate, and acetonitrile were by Carlo Erba (Milan, Italy). Distilled water was purified at 18.2 M $\Omega$ with a MilliQ ULTRA (Millipore, Vimodrone (MI), Italy) purification system. A mixture of dispersive SPE Citrate Extraction Tube Supelco ( $4 \mathrm{~g}$ magnesium sulphate, $1 \mathrm{~g}$ sodium chloride, $0.5 \mathrm{~g}$ sodium citrate dibasic sesquihydrate, and $1 \mathrm{~g}$ sodium citrate tribasic dihydrate) was used, supplied by Sigma-Aldrich (Milan, Italy).

2.2. Instrumentation. Ultra high-performance liquid chromatography UHPLC-MS/MS (Thermo Scientific, TSQ Quantum Access Max) equipped with Thermo hypersilgold column $(50 \mathrm{~mm} \times 2.1 \mathrm{~mm}, 1.9 \mu \mathrm{m})$ was used for quantification of neonicotinoids. The flow rate was $400 \mu \mathrm{L} / \mathrm{min}$, the column temperature $30^{\circ} \mathrm{C}$, and the injection loop volume $5 \mu \mathrm{L}$. A binary gradient of $0.05 \% \mathrm{HCOOH}$ and $\mathrm{HCOONH}_{4}$ $2 \mathrm{mM}$ in water (A) and $0.05 \% \mathrm{HCOOH}$ and $\mathrm{HCOONH}_{4}$ $2 \mathrm{mM}$ in $\mathrm{CH}_{3} \mathrm{OH}$ (B) was employed. The mobile-phase gradient was programmed as follows: $0 \mathrm{~min}, 10 \% \mathrm{~B} ; 7 \mathrm{~min}$, $95 \% \mathrm{~B} ; 8 \mathrm{~min}, 95 \% \mathrm{~B} ; 9 \mathrm{~min}, 10 \% \mathrm{~B}$; and $10 \mathrm{~min}, 10 \% \mathrm{~B}$. Mass spectral analyses were performed using an LC-TSQ Quantum Access Max operating in the positive ion mode
TABLE 1: UHPLC-MS/MS fragmentation of studied neonicotinoids.

\begin{tabular}{|c|c|c|c|c|}
\hline MW & Pesticide & $\begin{array}{l}\text { Precursor ion } \\
(m / z)\end{array}$ & $\begin{array}{l}\text { Product ions } \\
(m / z)\end{array}$ & $\begin{array}{c}\text { Collision } \\
\text { energy }(\mathrm{eV})\end{array}$ \\
\hline \multirow{3}{*}{222} & \multirow{3}{*}{ Acetamiprid } & \multirow{3}{*}{223.0} & 90.3 & 31 \\
\hline & & & 99.2 & 34 \\
\hline & & & 126.2 & 19 \\
\hline \multirow{3}{*}{249} & \multirow{3}{*}{ Clothianidin } & \multirow{3}{*}{250.0} & 113.2 & 25 \\
\hline & & & 132.1 & 16 \\
\hline & & & 169.2 & 12 \\
\hline \multirow{3}{*}{252} & \multirow{3}{*}{ Thiacloprid } & \multirow{3}{*}{253.0} & 90.3 & 36 \\
\hline & & & 99.2 & 37 \\
\hline & & & 126.2 & 21 \\
\hline \multirow{2}{*}{255} & \multirow{2}{*}{ Imidacloprid } & \multirow{2}{*}{256.0} & 175.2 & 17 \\
\hline & & & 209.1 & 14 \\
\hline \multirow{3}{*}{270} & \multirow{3}{*}{ Nitenpyram } & \multirow{3}{*}{271.1} & 126.2 & 27 \\
\hline & & & 225.2 & 9 \\
\hline & & & 237.2 & 21 \\
\hline \multirow{3}{*}{291} & \multirow{3}{*}{ Thiamethoxam } & \multirow{3}{*}{292.0} & 181.2 & 20 \\
\hline & & & 210.2 & 7 \\
\hline & & & 211.2 & 10 \\
\hline
\end{tabular}

using a h-ESI interface. The electrospray ionization (ESI) needle spray voltage was 4000 . The heated capillary was $270^{\circ} \mathrm{C}$. Flush volume was $700 \mu \mathrm{L}$ and Collision Gas Pressure was 1.3 mTorr. The neonicotinoids and the internal standard TCPP were detected in MS/MS conditions, programming the chromatographic run in SRM mode (selected reaction monitoring) as reported in Table 1. Preliminary tunings were carried out with continuous introduction of a dilute solution of certified standards. Flow rate of syringe pump infusion of $5 \mu \mathrm{L} / \mathrm{min}$ and the voltages on the lenses were optimized in TSQ Tune Master (Excalibur software).

2.3. Reference Solution. The standard mix solution at $5 \mu \mathrm{g} / \mathrm{mL}$ of standard pesticides was diluted by transferring $500 \mu \mathrm{L}$ $(100.0 \pm 0.5 \mu \mathrm{g} / \mathrm{mL})$ into a volumetric flask $(10 \mathrm{~mL}$, Class A certified).

The standard mix solution at $1 \mu \mathrm{g} / \mathrm{mL}$ of standard pesticides was diluted by transferring $100 \mu \mathrm{L}(100.0 \pm 0.5 \mu \mathrm{g} / \mathrm{mL})$ into a volumetric flask $(10 \mathrm{~mL}$, Class A certified).

The standard mix solution at $0.1 \mu \mathrm{g} / \mathrm{mL}$ of standard pesticides was diluted by transferring $200 \mu \mathrm{L}$ of solution at $5 \mu \mathrm{g} / \mathrm{mL}$ into a volumetric flask (10 mL, Class A certified). All mix solutions are making up at volume with acetonitrile. Stock solutions stored at $-18^{\circ} \mathrm{C}$ were stable for at least 3 months.

\subsection{Method Validation}

2.4.1. Specificity. The specificity of the analytical method for neonicotinoids detection was confirmed by obtaining positive results from honey containing the analyte, coupled with negative results from samples which do not contain 
it (negative controls). The matrix effect was assessed by preparing pesticide standards in blank matrix extracted from untreated honey. The matrix extracts were analyzed before spiking to confirm the absence of the test pesticides in them.

2.4.2. Linearity. The quantification of pesticide was based on a six-point matrix-matched calibration graph by plotting the detector response (SRM area ratio with respect to internal standard TCPP) against concentration of the calibration standards within the range 1-50 $\mu \mathrm{g} / \mathrm{L}$ making three replicates for each concentration. A linear regression of six calibration points for each component was used to determine the relationship with the analyte concentrations calculated for each component on the basis of their occurrence in the reference material. The regression equations with slope, $y$-intercept, and coefficient of correlation $\left(r^{2}\right)$ were evaluated for acetamiprid, clothianidin, imidacloprid, nitenpyram, thiacloprid, and thiamethoxam. Statistical test (Mandel and residual analysis with normal distribution of the calibration points) were performed to prove the linearity of regression lines.

2.4.3. Limit of Detection (LOD) and Limit of Quantification (LOQ). The LOD and LOQ were determined by signal-tonoise approach [14]. The noise and signal are measured experimentally on the chromatogram printout. LOQ was estimated by the response of method noise level by approximately ten and LOD is, therefore, 3.3 -fold lower.

\subsubsection{Method Accuracy (Recovery) and Precision (Repeata-} bility). Method recovery studies were performed at two spiking concentration levels $(10 \mu \mathrm{g} / \mathrm{kg}$ and $40 \mu \mathrm{g} / \mathrm{kg})$. The sample matrix was prepared by homogenizing a series of different honeys in order to develop a highly specific method. The samples were prepared by weighing $5.0 \pm 0.5 \mathrm{~g}$ of honey spiked in $50 \mathrm{~mL}$ tube (Meus srl, Piove di Sacco (PD), Italy). These sample tubes were vortexed (Velp, Usmate (MB), Italy) for 30 seconds after adding $10 \mathrm{~mL}$ of water and $10 \mathrm{~mL}$ of acetonitrile, in order to homogenize and fluidize the sample, and $50 \mu \mathrm{L}$ of Tris(1-chloro-2-propyl)phosphate (TCPP) at $50 \mathrm{mg} / \mathrm{L}$. In each tube was added a mixture of salts ( $4 \mathrm{~g}$ magnesium sulphate, $1 \mathrm{~g}$ sodium chloride, $0.5 \mathrm{~g}$ sodium citrate dibasic sesquihydrate, and $1 \mathrm{~g}$ sodium citrate tribasic dihydrate). The extract was stirred for 1 minute in vortex, in order to maximize the distribution of the analytes in the organic phase. The samples were centrifuged at $3000 \mathrm{rpm}$ for 5 minutes and the supernatant was filtered at $0.45 \mu \mathrm{m}$ PTFE filters (VWR, Milan, Italy). The extract was analyzed by UHPLC-MS/MS, making 6 replicates for each concentration. The average percentage of recovery and the relative standard deviation (RSD, repeatability) were evaluated.

2.4.5. Determination of Uncertainties. Combined uncertainty in estimation was determined for all the neonicotinoids at the two fortification levels studied ( 10 and $40 \mu \mathrm{g} / \mathrm{kg}$ ) as the statistical procedure of the EURACHEM/CITAC Guide CG 4 [15]. Individual sources of uncertainty were taken into account as described below.
Uncertainty of Analytical Standard Solutions. As the uncertainty of standard concentration declared in the supplier's certificate was given without any confidence level, rectangular distribution was assumed for calculating standard uncertainty

$$
U 1=\frac{u(x) / C(x)}{\sqrt{ } 3},
$$

where $u(x)$ represents the uncertainty value given in the certificate and $C(x)$ the concentration of the standard solution.

Uncertainty of Weighing. The relative uncertainty due to honey weighing was calculated using normal distribution given by

$$
U 2=\frac{(0.00005)}{W i},
$$

where $W i$ is the weight of the sample, and 0.00005 is the value of uncertainty of the balance at $95 \%$ confidence level as reported in the certificate.

Uncertainty of Calibration Linearity. Uncertainty associated with the calibration curve, was calculated according to

$$
U 3=\left(\frac{s}{b_{1}}\right)\left(\left\{\frac{1}{p}\right\}+\left\{\frac{1}{n}\right\}+\left\{\frac{\left(c_{0}-c^{\prime}\right)^{2}}{s_{x x}}\right\}\right)^{1 / 2},
$$

where $s$ is the standard deviation of the residuals of the calibration curve, $b_{1}$ is the slope of the calibration curve, $p$ is the number of measurements of the unknown, $n$ is the number of points used to form the calibration curve, $c_{0}$ is the calculated concentration of the analyte from the calibration curve, $c^{\prime}$ is the arithmetic mean of the concentrations of the standards used to make the calibration curve, and $s_{x x}$ is calculated as given in

$$
s_{x x}=\sum\left(c j-c^{\prime}\right)^{2},
$$

where $j=1,2, \ldots, n$. $c j$ is the concentration of each calibration standard used to build up the calibration curve.

Uncertainty Associated with Precision. In the present study, the random errors of extraction, clean up, and UHPLC analyses steps were approximated by standard deviations which were calculated from repeated determinations of analytes expressed as repeatability. The precision was calculated according to

$$
U 4=\frac{s}{(\sqrt{ } n \times x)},
$$

where $s$ is the standard deviation of the results obtained from the recovery study, $n$ is the number of assays and $x$ is the mean value of the concentration recovered.

Uncertainty of Volume. The volume of the solution is subject to 3 sources of uncertainty: calibration, repeatability, and temperature effects. (a) Calibration: the uncertainty in the certified internal volume of the flask and of the pipettes. For 


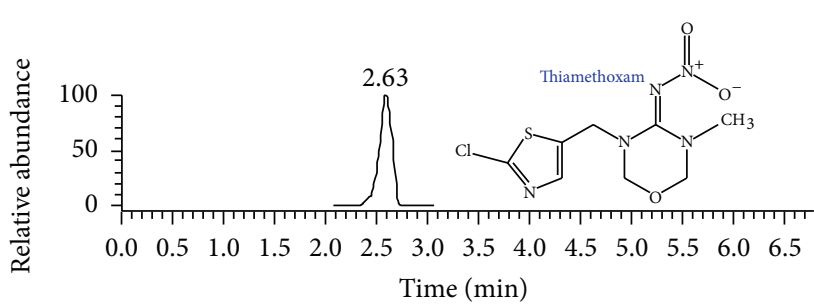

(a)

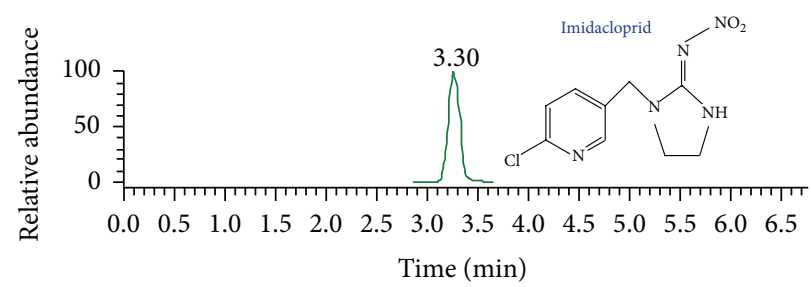

(c)

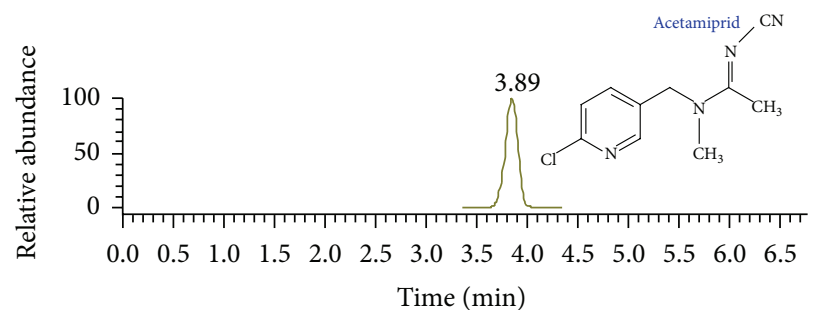

(e)

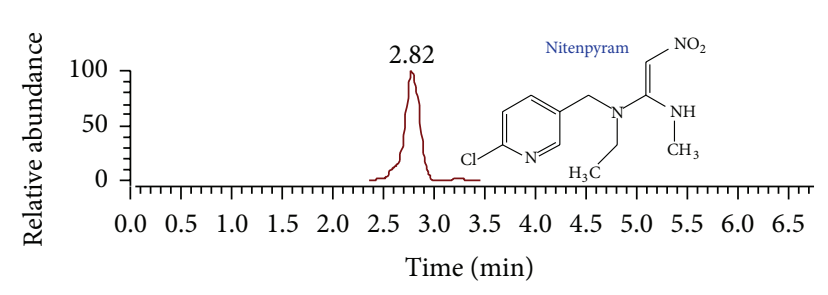

(b)

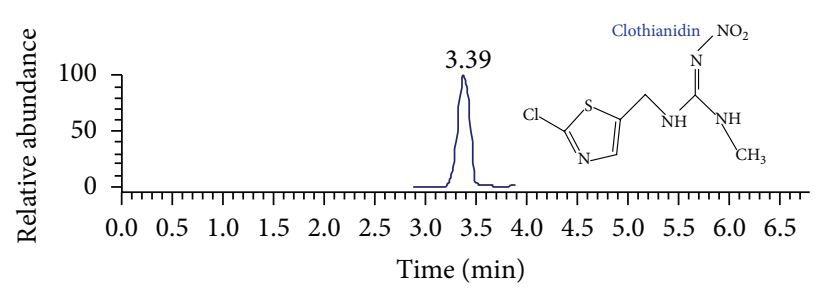

(d)

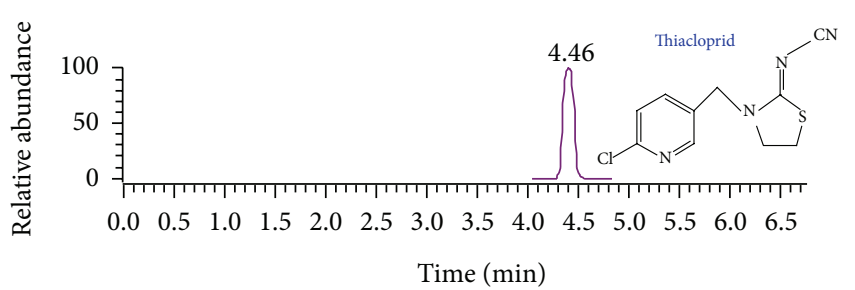

(f)

FIgURE 1: Representative UHPLC/MS-MS chromatogram of studied neonicotinoids for matrix-matched standards $40 \mu \mathrm{g} / \mathrm{kg}$.

example, the manufacturer gives a volume of $10.00 \pm 0.02 \mathrm{~mL}$ $(V \pm a)$ for the flask, when measured at a temperature of $20^{\circ} \mathrm{C}$. Because the value of the uncertainty is given without a confidence level or distribution information, an assumption is necessary. In this work, the standard uncertainty is calculated by assuming a triangular distribution according to

$$
U 5=\frac{(a / \sqrt{ } 3)}{V} .
$$

In the same way, the volumes of the pipettes used to prepare the solutions at different levels are calculated by assuming a triangular distribution. The contributions due to the dilution operations performed for each concentration level are calculated separately and combined to give the standard uncertainty of the volume. (b) Repeatability: the uncertainty due to variations in filling is considered in the repeatability experiments. (c) Temperature: the temperatures of the flask and solution differ from the temperature at which the volume of the flask was calibrated. According to the manufacturer, the flask was calibrated at a temperature of $20^{\circ} \mathrm{C}$, whereas the laboratory temperature varies by $\pm 2^{\circ} \mathrm{C}$. The uncertainty from this effect can be calculated from the estimate of the temperature range and the coefficient of the volume expansion. In the case of acetonitrile as a solvent, this effect is negligible.
The combined uncertainty $(U)$ was calculated as $U=$ $x\left[\left(U 1^{2}+U 2^{2}+U 3^{2}+U 4^{2}\right)^{1 / 2}\right]$, where $C x$ is the mean neonicotinoids concentration, and reported as expanded uncertainty $(2 U)$ which is twice the value of the combined uncertainty at $95 \%$ confidence level.

\section{Results and Discussion}

3.1. Method Development. In order to identify the major species produced in collisional experimental fragmentation of MS/MS analysis, a mass characterization study was firstly performed for direct infusion of each investigated neonicotinoids. Mass scans in positive ions mode were performed with h-ESI source ionization; all investigated molecules showed a good fragmentation. The collision energy was modulated from 5 to 50 of instrumental maximum to obtain the better fragmentation pattern. The ESI spectrum is characterized by the parent ion $[\mathrm{M}+\mathrm{H}]^{+}$for all molecules. The neutral losses of $\mathrm{NO}_{2}$ and/or $\mathrm{HCl}$ were observed for clothianidin, imidacloprid, nitenpyram, and thiamethoxam. The fragment at $\mathrm{m} / z 126$, corresponding to $\left[\mathrm{C}_{6} \mathrm{H}_{5}-\mathrm{OCl}\right]^{+}$was a characteristic for acetamiprid, nitenpyram, and thiacloprid (Table 1). The discussed SRM data were in agreement with what reported by Sabatino et al. [10] and Ferrer et al. [16]. 
TABLE 2: Method validation results.

\begin{tabular}{lcccccc}
\hline Compound & $R_{t}(\mathrm{~min})$ & Linearity range $(\mu \mathrm{g} / \mathrm{L})$ & $r^{2}$ & LOQ $(\mu \mathrm{g} / \mathrm{kg})$ & Recovery $\%(10 \mu \mathrm{g} / \mathrm{kg})^{\dagger}$ & Recovery \% $(40 \mu \mathrm{g} / \mathrm{kg})^{\dagger}$ \\
\hline Thiamethoxam & 2.63 & $1-50$ & 0.999 & 0.50 & $101 \pm 11$ & $100 \pm 12$ \\
Nitenpyram & 2.82 & $1-50$ & 0.998 & 4.00 & $75 \pm 20$ & $97 \pm 9$ \\
Imidacloprid & 3.30 & $1-50$ & 0.998 & 2.80 & $114 \pm 3$ & $109 \pm 7$ \\
Clothianidin & 3.39 & $1-50$ & 0.999 & 3.20 & $111 \pm 8$ & $105 \pm 9$ \\
Acetamiprid & 3.89 & $1-50$ & 0.995 & 0.12 & $107 \pm 5$ & $105 \pm 6$ \\
Thiacloprid & 4.46 & $1-50$ & 0.997 & 0.10 & $89 \pm 6$ & $92 \pm 2$ \\
\hline
\end{tabular}

${ }^{\dagger}$ Mean value of six determinations; relative standard deviations (precision) in parenthesis.

The chromatographic method has been developed on the results of preliminary studies carried out on matrixfortified standards. Different solvents were used for the chromatographic separation and several chromatographic separations were evaluated. The best results were obtained using an elution gradient starting with a binary gradient of $0.05 \% \mathrm{HCOOH}$ and $\mathrm{HCOONH}_{4} 2 \mathrm{mM}$ in water and

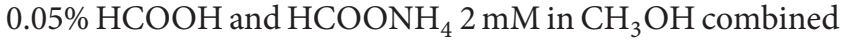
with the Thermo hypersil GOLD $50 \times 2.1 \mathrm{~mm}(1.9 \mu \mathrm{m}$ i.d. $)$ column. Under the described chromatographic conditions, the studied molecules were resolved in less than 5 minutes (Figure 1) and well recognizable on the basis of $m / z$ signals, and good sensitivities were obtained; each analyte showed a typical mass spectrum profile previously identified by direct infusion.

The concept of a single extraction and dilution of the extracts was chosen in this study to achieve good results in the shortest time. In 2011, Tanner and Czerwenka [11] applied two steps of purification with d-SPE applying the QuEChERS methodology to the honey. Our protocol eliminated the second purification step, limiting the extraction to the use of d-SPE citrate extraction tube and reducing times and costs of analyses. Nevertheless, results were satisfactory in terms of statistical parameters, the selectivity for the analytes of interest, and reduction of the matrix effect (see paragraph below). This protocol permitted to analyze a high number of samples per day and is, therefore, suitable for a routine application in control laboratories. The proposed analytical protocol is currently applied in ICQRF Catania laboratory in the frame of Italian Ministry quality control investigation.

3.2. Method Validation. Analytical parameters of the proposed method were evaluated according to the criteria given in Section 2. Results are reported in Table 2.

3.2.1. Specificity. The specificity of the method toward the studied analytes was good. No interferences due to matrixes were found. Hence, no further time-consuming concentration/cleanup pretreatments were required.

3.2.2. Linearity of Calibration Curve. The linearity of each pesticide was established by plotting UHPLC response area ratio versus concentration. The analytes showed linear behavior in the studied concentration range of $1-50 \mu \mathrm{g} / \mathrm{L}$. The correlation coefficient $\left(r^{2}\right)$ was found to be $\geq 0.995$ for all pesticides.
3.2.3. $L O D$ and LOQ. LOD and LOQ were estimated as the lowest concentrations of pesticide injected that yielded a signal/noise ratio of 3 and 10, respectively. LOQs evaluation showed the lowest value $0.10 \mu \mathrm{g} / \mathrm{kg}$ for thiacloprid to the higher value of $4.00 \mu \mathrm{g} / \mathrm{kg}$ for nitenpyram. The LOQs attained in the proposed method fit with maximum residue limits (MRLs) of $10 \mu \mathrm{g} / \mathrm{kg}$ for nonallowed pesticides [17].

3.2.4. Recovery and Precision. The single-step extraction method adopted for honey samples provided satisfactory recovery which ranged from $75 \%$ (nitepyram) to $114 \%$ (imidacloprid) for the fortification level of $10 \mu \mathrm{g} / \mathrm{kg}$ and from 92 (thiacloprid) to $109 \%$ (imidacloprid) for the fortification level of $40 \mu \mathrm{g} / \mathrm{kg}$. The precision of the method was good, not exceeding a coefficient of variation of $12 \%$, with the exception of nitenpyram at the lowest fortification level. These data are in agreement with the criteria of document no. SANCO/12495/2011, that recommend general recovery limits of $70-120 \%$ within laboratory repeatability $\leq 20 \%$ [18]. Therefore, the method could be considered sufficiently accurate and precise for the purpose.

3.2.5. Uncertainty of Measurement. The study of uncertainty was performed at 2 concentration levels (10 and $40 \mu \mathrm{g} / \mathrm{kg}$ ), identifying and studying the most important parameters that determined the uncertainty of the analytical method. The parameters selected were point calibration, standard solution, weigh, volume, and precision; their contributions to method uncertainty were calculated as indicated in the experimental section. The different contributions of uncertainty for each concentration level, together with the relative combined standard uncertainty, are shown in Tables 3 and 4 for each neonicotinoid. Results showed that the contribution to uncertainty due to the dilution operations and the standard purities was constant for each concentration level and for each analyte. The same value of uncertainty concerning the amount of weighed sample was used for each level and for all pesticides because the quantity of analyzed sample did not change among the experiments; moreover, this contribution could be considered negligible. The uncertainty associated with repeatability has a moderate contribution to the expanded uncertainties, showing the higher value for nitenpyram, thiamethoxam, and clothianidin. The $10 \mu \mathrm{g} / \mathrm{kg}$ level showed the uncertainty of calibration point as the main constituent of total uncertainty, followed by the volume contribution. On the contrary, the volume uncertainty was 
TABLE 3: Results of individual and combined uncertainties for each pesticide calculated at $10 \mu \mathrm{g} / \mathrm{kg}$ concentration level.

\begin{tabular}{|c|c|c|c|c|c|c|c|c|}
\hline Compound & $\begin{array}{c}\text { Standard } \\
\text { solution } \\
U 1\end{array}$ & $\begin{array}{c}\text { Weighting } \\
\text { U2 }\end{array}$ & $\begin{array}{c}\text { Calibration } \\
\text { curve } \\
\text { U3 }\end{array}$ & $\begin{array}{c}\text { Precision } \\
\text { U4 }\end{array}$ & $\begin{array}{c}\text { Dilution } \\
\text { operations } \\
\text { U5 }\end{array}$ & $\begin{array}{c}\text { Combined } \\
\text { uncertainty } \\
U\end{array}$ & $\begin{array}{c}\text { Expanded } \\
\text { uncertainty } \\
2 U\end{array}$ & $\begin{array}{c}\text { Relative expanded } \\
\text { uncertainty } \\
U \%\end{array}$ \\
\hline Thiamethoxam & 0.003 & 0.00001 & 0.064 & 0.044 & 0.070 & 1.1 & 2.2 & 21 \\
\hline Nitenpyram & 0.003 & 0.00001 & 0.164 & 0.082 & 0.070 & 1.5 & 3.0 & 39 \\
\hline Imidacloprid & 0.003 & 0.00001 & 0.161 & 0.011 & 0.070 & 2.0 & 4.0 & 35 \\
\hline Clothianidin & 0.003 & 0.00001 & 0.089 & 0.033 & 0.070 & 1.3 & 2.6 & 23 \\
\hline Acetamiprid & 0.003 & 0.00001 & 0.232 & 0.019 & 0.070 & 2.6 & 5.2 & 49 \\
\hline Thiacloprid & 0.003 & 0.00001 & 0.220 & 0.024 & 0.070 & 2.1 & 4.2 & 46 \\
\hline
\end{tabular}

TABLE 4: Results of individual and combined uncertainties for each pesticide calculated at $40 \mu \mathrm{g} / \mathrm{kg}$ concentration level.

\begin{tabular}{|c|c|c|c|c|c|c|c|c|}
\hline Compound & $\begin{array}{c}\text { Standard } \\
\text { solution } \\
U 1\end{array}$ & $\begin{array}{c}\text { Weighting } \\
\text { U2 }\end{array}$ & $\begin{array}{c}\text { Calibration } \\
\text { curve } \\
\text { U3 }\end{array}$ & $\begin{array}{c}\text { Precision } \\
\text { U4 }\end{array}$ & $\begin{array}{c}\text { Dilution } \\
\text { operations } \\
\text { U5 }\end{array}$ & $\begin{array}{c}\text { Combined } \\
\text { uncertainty } \\
U\end{array}$ & $\begin{array}{c}\text { Expanded } \\
\text { uncertainty } \\
2 U\end{array}$ & $\begin{array}{c}\text { Relative expanded } \\
\text { uncertainty } \\
U \%\end{array}$ \\
\hline Thiamethoxam & 0.003 & 0.00001 & 0.017 & 0.040 & 0.070 & 3.3 & 6.6 & 16 \\
\hline Nitenpyram & 0.003 & 0.00001 & 0.036 & 0.032 & 0.070 & 3.3 & 6.6 & 16 \\
\hline Imidacloprid & 0.003 & 0.00001 & 0.040 & 0.029 & 0.070 & 3.7 & 7.4 & 17 \\
\hline Clothianidin & 0.003 & 0.00001 & 0.023 & 0.037 & 0.070 & 3.5 & 7.0 & 16 \\
\hline Acetamiprid & 0.003 & 0.00001 & 0.061 & 0.023 & 0.070 & 4.0 & 8.0 & 19 \\
\hline Thiacloprid & 0.003 & 0.00001 & 0.054 & 0.009 & 0.070 & 3.3 & 6.6 & 17 \\
\hline
\end{tabular}

the major source to total uncertainty at the $40 \mu \mathrm{g} / \mathrm{kg}$ level, while the uncertainty of repeatability and calibration point had approximately similar values.

When the uncertainty of the result is reported, the combined standard uncertainty is multiplied with a so-called coverage factor, yielding an expanded uncertainty. A factor $k=2$ was used because of the resemblance of the expanded uncertainty to a $95 \%$ confidence interval. The document no. SANCO/12495/2011 recommended a default expanded uncertainty of $50 \%$ to be used by regulatory authorities in cases of enforcement decisions (MRL exceedances) [18]. Our results showed a relative uncertainty $(U \%)$ ranging from 21 (thiamethoxam) to $49 \%$ (acetamiprid) at levels of $10 \mu \mathrm{g} / \mathrm{kg}$. Lower values were obtained for the $40 \mu \mathrm{g} / \mathrm{kg}$ level. At this level, all pesticides had $U \%$ ranging from 16 to $19 \%$.

\section{References}

[1] S. Kagabu, "Studies on the synthesis and insecticidal activity of neonicotinoid compounds," Journal of Pesticide Science, vol. 21, pp. 237-239, 1996.

[2] K. Matsuda, S. D. Buckingham, D. Kleier, J. J. Rauh, M. Grauso, and D. B. Sattelle, "Neonicotinoids: insecticides acting on insect nicotinic acetylcholine receptors," Trends in Pharmacological Sciences, vol. 22, no. 11, pp. 573-580, 2001.

[3] T. Iwasa, N. Motoyama, J. T. Ambrose, and R. M. Roe, "Mechanism for the differential toxicity of neonicotinoid insecticides in the honey bee, Apis mellifera," Crop Protection, vol. 23, no. 5, pp. 371-378, 2004.

[4] Italian Government, 2008, Ministerial Decree of 17. 09. 2008 relating the precautionary suspension of the authorization of plant protection products containing active substances clothianidin, thiamethoxam, imidacloprid and fipronil for use in the seeds tanning. Italian Republic Official Gazzette of 20. 09. 2008 No. 221.

[5] Italian Government, 2012, Ministerial Decree of 25 giugno 2012 relating the prorogation of precautionary suspension of the authorization of plant protection products containing active substances clothianidin, thiamethoxam, imidacloprid and fipronil for use in the seeds tanning. Italian Republic Official Gazzette of 30.06.2012 No. 151.

[6] P. Fidente, S. Seccia, F. Vanni, and P. Morrica, "Analysis of nicotinoid insecticides residues in honey by solid matrix partition clean-up and liquid chromatography-electrospray mass spectrometry," Journal of Chromatography A, vol. 1094, no. 1-2, pp. 175-178, 2005.

[7] A. Kamel, "Refined methodology for the determination of neonicotinoid pesticides and their metabolites in honey bees and bee products by liquid chromatography-tandem mass spectrometry (LC-MS/MS)," Journal of Agricultural and Food Chemistry, vol. 58, no. 10, pp. 5926-5931, 2010.

[8] N. O. Z. Abaga, P. Alibert, S. Dousset, P. W. Savadogo, M. Savadogo, and M. Sedogo, "Insecticide residues in cotton soils of Burkina Faso and effects of insecticides on fluctuating asymmetry in honey bees (Apis mellifera Linnaeus)," Chemosphere, vol. 83, no. 4, pp. 585-592, 2011.

[9] S. Rossi, A. G. Sabatini, R. Cenciarini, S. Ghini, and S. Girotti, "Use of high-performance liquid chromatography-UV and gas chromatography-mass spectrometry for determination of the imidacloprid content of honeybees, pollen, paper filters, grass, and flowers," Chromatographia, vol. 61, no. 3-4, pp. 189-195, 2005.

[10] L. Sabatino, M. Scordino, V. Pantò, E. Chiappara, P. Traulo, and G. Gagliano, "Survey of neonicotinoids and fipronil in corn seeds for agriculture," Food Additives and Contaminants B, no. 1, pp. 11-16, 2012. 
[11] G. Tanner and C. Czerwenka, "LC-MS/MS analysis of neonicotinoid insecticides in honey: methodology and residue findings in Austrian honeys," Journal of Agricultural and Food Chemistry, vol. 59, no. 23, pp. 12271-12277, 2011.

[12] S. Totti, M. Fernández, S. Ghini et al., "Application of matrix solid phase dispersion to the determination of imidacloprid, carbaryl, aldicarb, and their main metabolites in honeybees by liquid chromatography-mass spectrometry detection," Talanta, vol. 69, no. 3, pp. 724-729, 2006.

[13] M. Anastassiades, S. J. Lehotay, D. Štajnbaher, and F. J. Schenck, "Fast and easy multiresidue method employing acetonitrile extraction/partitioning and "dispersive solid-phase extraction" for the determination of pesticide residues in produce," Journal of AOAC International, vol. 86, no. 2, pp. 412-431, 2003.

[14] J. Vial and A. Jardy, "Experimental comparison of the different approaches to estimate LOD and LOQ of an HPLC method," Analytical Chemistry, vol. 71, no. 14, pp. 2672-2677, 1999.

[15] EURACHEM/CITAC Guide CG 4, EURACHEM/CITAC Guide (2000), Quantifying uncertainty in analytical measurement, 2nd edition, http://www.measurementuncertainty.org.

[16] I. Ferrer, E. M. Thurman, and A. M. Fernàndez-Alba, "Quantitation and accurate mass analysis of pesticides in vegetables by LC/TOF-MS," Analytical Chemistry, vol. 77, no. 9, pp. 28182825, 2005.

[17] European Commission Regulation (EC), “No. 396/2005 of 23 February 2005 on maximum residue levels of pesticides in or on food and feed of plant and animal origin and amending Council Directive 91/414/EEC," Official Journal of the European Union, vol. L70, pp. 1-16.

[18] Document No. SANCO/12495/2011, Method Validation and Quality Control Procedures for Pesticide Residues Analysis in Food and Feed, http://ec.europa.eu/food/plant/protection/pesticides/docs/qualcontrol_en.pdf. 

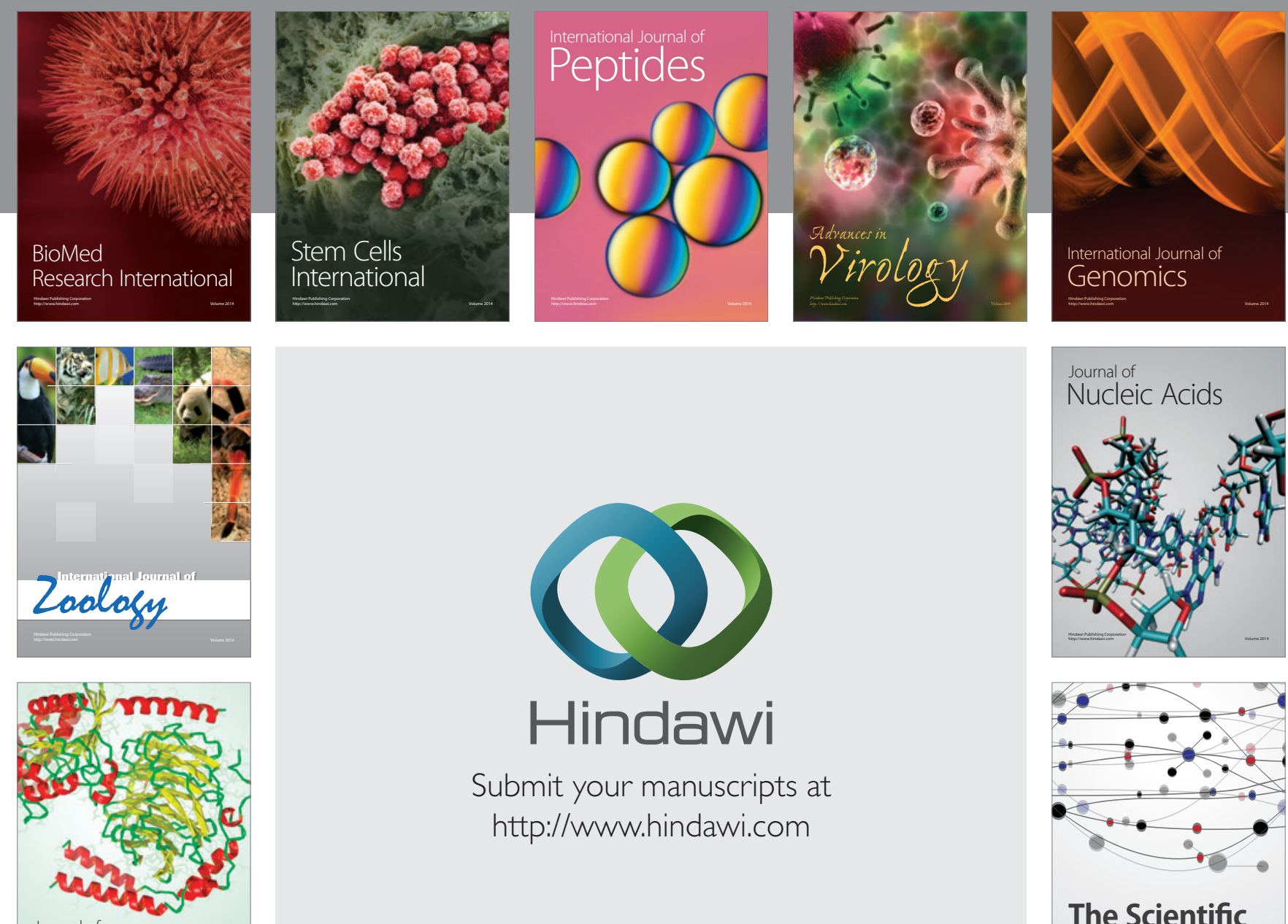

Submit your manuscripts at

http://www.hindawi.com

Journal of
Signal Transduction
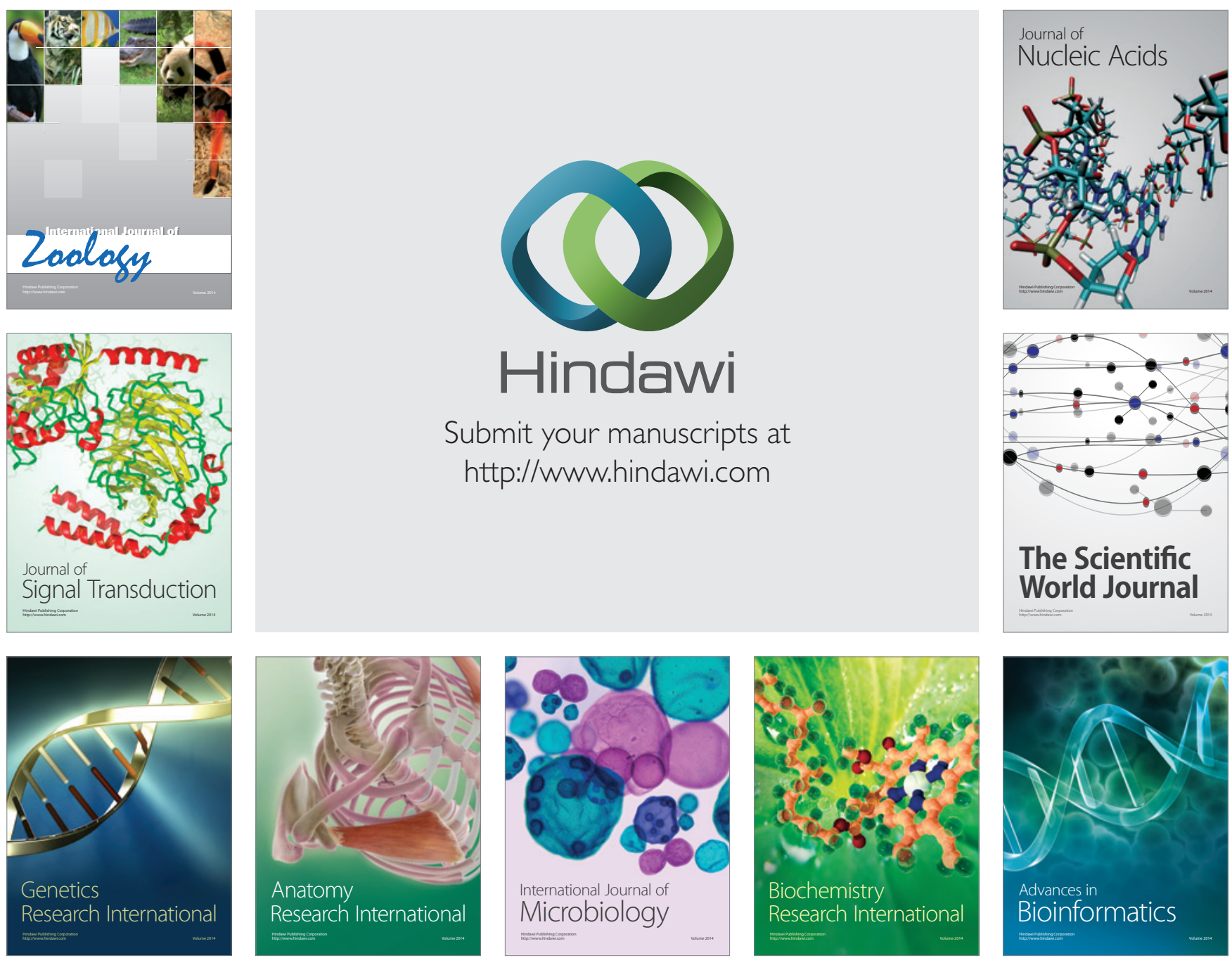

The Scientific World Journal
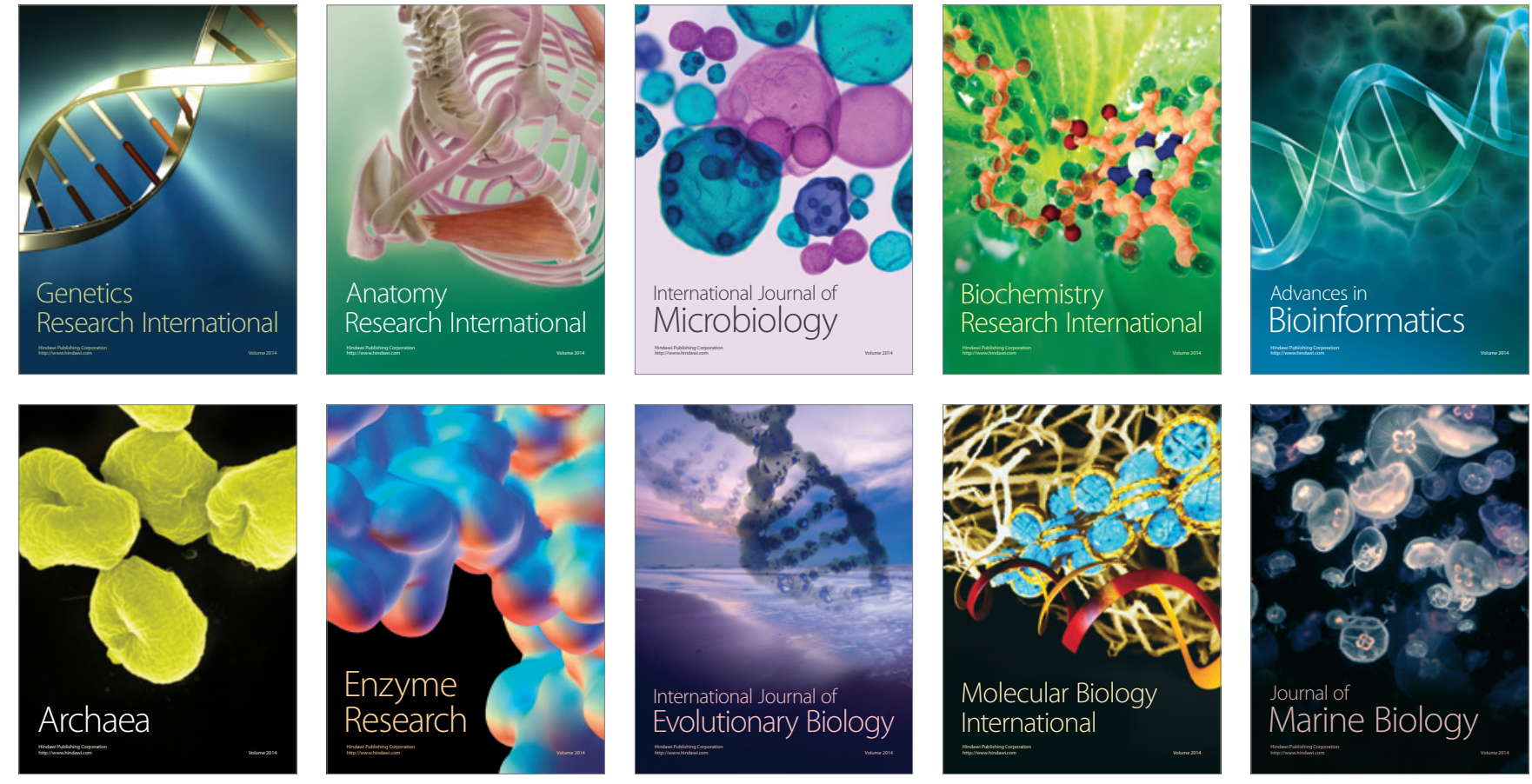